\title{
Blow-up of Electric Fields between Closely Spaced Spherical Perfect Conductors
}

\author{
Mikyoung Lim* KiHyun Yun ${ }^{\dagger}$
}

October 28, 2018

\begin{abstract}
The electric field increases toward infinity in the narrow region between closely adjacent perfect conductors as they approach each other. Much attention has been devoted to the blow-up estimate, especially in two dimensions, for the practical relevance to high stress concentration in fiber-reinforced elastic composites. In this paper, we establish optimal estimates for the electric field associated with the distance between two spherical conductors in $n$ - dimensional spaces for $n \geq 2$. The novelty of these estimates is that they explicitly describe the dependency of the blow-up rate on the geometric parameters: the radii of the conductors.
\end{abstract}

MSC-class: 15A15, 15A09, 15A23

\section{Introduction}

We consider the blow-up of the electric fields in the narrow region between a pair of perfect conductors which is closely adjacent in $n$ dimensions $(n \geq 2)$. Conductors provide higher intensity of electric flux around them. The intensity increases as a pair of conductors approaches each other, and the electric field even reaches toward infinity (refer to [2, 3, 11, 12, 1, 4)).

In this paper, we present the optimal blow-up estimate for the electric field with respect to the distance between a pair of conductors under the assumption that the conductors are of spherical shape in $n$ dimensions $(n \geq 2)$. The novelty of these estimate is to describe explicitly the dependency of the blow-up rate on the radii of the conductors: this paper is the first result to establish the role of the geometrical factor of conductors in the blow-up of the electric field in three or higher dimensions.

Besides the consideration of the gradient estimates in the frame of the electrostatic theory, much attention has been drawn to it of the relevance to the stress-strain behavior of composite materials, especially in two dimensions. According to Budiansky and Carrier

\footnotetext{
*Department of Mathematics, Colorado State University, Fort Collins, CO 80523, USA(lim@math.colostate.edu)

${ }^{\dagger}$ Department of Mathematics, Michigan State University, East Lansing, MI 48824, USA(kyun@math.msu.edu)
} 
[5], unexpectedly low strengths in stiff fiber-reinforced composites have been reported, due to the high stress concentration occurring in the narrow region between fibers (also refer to [8]). In the anti-plane shear model, the stress tensor represents the electric field in the two dimensional conductivity model, where the out-of-plane elastic displacement satisfies a conductivity equation [6]. Thus, the gradient estimates for electric field have a valuable meaning in relation to in the failure analysis of composite material. To give a brief description of related works, for the case that the inclusions and the outside of inclusions have the comparable conductivities (or shear moduli), it was verified that the electric field remains bounded independently of the distance between the inclusions. Li and Vogelius [10] have shown that the electric field does not blow up even when the inclusions approaches each other. Moreover, Li and Nirenberg 9] have extended this result to elliptic systems. These results point out that the extremely high conductivity (or the stiffness of fibers) is indispensable to the blow-up phenomena.

In this respect, much attention has been focused on the model of a pair of perfect conductors which are $\epsilon$ apart. Ammari, Kang, H. Lee, J. Lee and Lim 2, 3] have established the optimal blow-up rate $\epsilon^{-1 / 2}$ as the distance $\epsilon$ goes to zero, when conductors are of circular shape in two dimensions. Yun [11, 12] has extended the above mentioned result to a sufficiently general class of the conductors' shapes in two dimensions. In three or higher dimensional case, Bao, Li and Yin 4 recently obtained the optimal blow-up rate for perfect conductors of general shape: the optimal blow-up rate is $(\epsilon|\log \epsilon|)^{-1}$ for three dimensions, and is $\epsilon^{-1}$ for higher $n$ dimensions $(n \geq 4)$. However, their estimates are only given by the distance between two conductors and geometric information of conductors are not incorporated into the estimates.

What is new in this paper is that for the case of spherical perfect conductors in three and higher dimensions, the gradient estimates are established in terms of the radii as well as the distance between inclusions. What is more is that the approach introduced in this paper to derive the estimates is distinct from the methods of Bao et al. [4 and Ammari et al. 2, 3. In the two dimensional case, our approach provides the same estimates as of Ammari et el., Proposition 3.2 in a much simpler way for the case of perfect inclusions.

\section{Mathematical formalism and main results}

From now on, $\mathbb{R}^{n}$ denotes $n$ dimensions, and $B_{r}\left(x_{1}, x_{2}, \cdots, x_{n}\right)$ is the sphere with radius $r$ and center $\left(x_{1}, x_{2}, \cdots, x_{n}\right)$ in $\mathbb{R}^{n}$. Given any entire harmonic function $H$ in $\mathbb{R}^{n}(n \geq 2)$, we define the electric potential $u$ as the unique solution to the following conductivity problem:

$$
\begin{cases}\Delta u=0, & \text { in } \mathbb{R}^{n} \backslash \overline{\left(D_{1} \cup D_{2}\right)}, \\ u(\mathbf{x})-H(\mathbf{x})=O\left(|\mathbf{x}|^{1-n}\right) & \text { as }|\mathbf{x}| \rightarrow \infty, \\ \left.u\right|_{\partial D_{i}}=C_{i}(\text { constant }), & \text { for } i=1,2, \\ \int_{\partial D_{i}} \partial_{\nu} u d S=0, & \end{cases}
$$

where $\mathbf{x}=\left(x_{1}, x_{2}, \cdots, x_{n}\right)$. This solution $u$ can be interpreted physically as the electric potential outside conductors $D_{1}$ and $D_{2}$ under the action of applied electric field $\nabla H$.

In this paper, we start by considering the case that $\nabla H$ is a uniform field, i.e, $H=\mathbf{a} \cdot \mathbf{x}$ for some constant $\mathbf{a}$ in $\mathbb{R}^{n}$, in Theorem 2.1 and 2.3. Based on these, the optimal upper bound of the gradient for any entire harmonic function $H$ is established in Theorem 2.4. 
Theorem 2.1 (Three dimensions) We assume that $D_{1}$ and $D_{2}$ are the pair of spheres with radii $r_{1}$ and $r_{2}$ that are $2 \epsilon$ apart in $\mathbb{R}^{3}$. Thus, we set

$$
D_{1}=B_{r_{1}}\left(r_{1}+\epsilon, 0,0\right) \text { and } D_{2}=B_{r_{2}}\left(-\left(r_{2}+\epsilon\right), 0,0\right) .
$$

Let $u$ be the solution to (1) for $H\left(x_{1}, x_{2}, x_{3}\right)=\sum_{i=1}^{3} a_{i} x_{i}$. Then, there exists a positive constant $C_{*}$ independent of $\epsilon, r_{1}, r_{2}$ and $\left(a_{1}, a_{2}, a_{3}\right)$ such that

$$
\frac{1}{C_{*}}\left|a_{1}\right|\left(\frac{r_{1} r_{2}}{r_{1}+r_{2}}\right) \frac{1}{|\log \epsilon|} \leq|u|_{\partial D_{1}}-\left.u\right|_{\partial D_{2}}\left|\leq C_{*}\right| a_{1}\left|\left(\frac{r_{1} r_{2}}{r_{1}+r_{2}}\right)\right| \log \epsilon \mid
$$

for sufficiently small $\epsilon>0$.

(a) In the case that $a_{1}$ is nonzero, for any sufficiently small $\epsilon$, there is a point $\mathbf{x}_{0}$ between $D_{1}$ and $D_{2}$ such that

$$
\frac{1}{2 C_{*}}\left|a_{1}\right|\left(\frac{r_{1} r_{2}}{r_{1}+r_{2}}\right) \frac{1}{|\epsilon \log \epsilon|} \leq\left|\nabla u\left(\mathbf{x}_{0}\right)\right| .
$$

The lower bound above is optimal in the sense that there is a positive constant $C^{*}$ independent of $\epsilon, r_{1}, r_{2}$ and $\left(a_{1}, a_{2}, a_{3}\right)$, satisfying that

$$
\|\nabla u\|_{L^{\infty}\left(\mathbb{R}^{3} \backslash\left(D_{1} \cup D_{2}\right)\right)} \leq C^{*}\left|a_{1}\right|\left(\frac{r_{1} r_{2}}{r_{1}+r_{2}}\right) \frac{1}{|\epsilon \log \epsilon|},
$$

for sufficiently small $\epsilon>0$.

(b) In the case that $a_{1}$ is zero, the gradient of $u$ does not blow up even when the distance $\epsilon$ goes to zero, i.e., there is a positive constant $C_{0}^{*}$ independent of $\epsilon$ and $\left(a_{1}, a_{2}, a_{3}\right)$, satisfying that

$$
\|\nabla u\|_{L^{\infty}\left(\mathbb{R}^{3} \backslash\left(D_{1} \cup D_{2}\right)\right)} \leq C_{0}^{*}\left(\left|a_{2}\right|+\left|a_{3}\right|\right),
$$

for sufficiently small $\epsilon>0$.

Remark 2.2 The constant $C_{0}^{*}$ at (3) depends on $r_{1}$ and $r_{2}$ : in details, there is a constant $C$ so that

$$
\|\nabla u\|_{L^{\infty}\left(\mathbb{R}^{3} \backslash\left(D_{1} \cup D_{2}\right)\right)} \leq C \max \left\{\frac{r_{1}}{r_{2}}, \frac{r_{2}}{r_{1}}\right\}\left(\left|a_{2}\right|+\left|a_{3}\right|\right),
$$

when $a_{1}=0$. The derivation of the inequality above is included in the proof of Theorem 2.1. The term 'C $\max \left\{\frac{r_{1}}{r_{2}}, \frac{r_{2}}{r_{1}}\right\}$ ' becomes arbitrarily large for small $r_{1}$ or $r_{2}$, and then it is not guaranteed that the bound (44) above is optimal. However, our attention is focused on the contribution of $r_{1}$ and $r_{2}$ to the blow-up rate associated to the distance $2 \epsilon$. As mentioned in Theorem 2.1, the gradient of $u$ blows up as $\epsilon \rightarrow 0$ if and only if $a_{1}$ is nonzero. On this occasion, the $1 /|\epsilon \log \epsilon|$ term at (2) dominates the upper bound of $\|\nabla u\|_{L^{\infty}\left(\mathbb{R}^{3} \backslash\left(D_{1} \cup D_{2}\right)\right)}$, and the bound (2) describes the contribution of $r_{1}$ and $r_{2}$ well. Therefore, Theorem 2.1 would suffices for our purpose.

Theorem 2.3 (Higher dimensions) We assume that $D_{1}$ and $D_{2}$ are the pair of spheres with radii $r_{1}$ and $r_{2}$ that are $2 \epsilon$ apart in $\mathbb{R}^{n}(n \geq 4)$. Thus, we set

$$
D_{1}=B_{r_{1}}\left(r_{1}+\epsilon, 0, \cdots, 0\right) \text { and } D_{2}=B_{r_{2}}\left(-\left(r_{2}+\epsilon\right), 0, \cdots, 0\right) \text {. }
$$


Let $u$ be the solution to (1) for $H\left(x_{1}, x_{2}, \cdots, x_{n}\right)=\sum_{i=1}^{n} a_{i} x_{i}$. Then, there exists a constant $C_{* *}$ independent of $\epsilon, r_{1}, r_{2}$ and $\left(a_{1}, a_{2}, \cdots, a_{n}\right)$ such that

$$
\frac{1}{C_{* *}}\left|a_{1}\right|\left(\frac{r_{1} r_{2}}{r_{1}+r_{2}}\right) \leq|u|_{\partial D_{1}}-\left.u\right|_{\partial D_{2}}\left|\leq C_{* *}\right| a_{1} \mid\left(\frac{r_{1} r_{2}}{r_{1}+r_{2}}\right)
$$

for sufficiently small $\epsilon>0$.

(a) In the case that $a_{1}$ is nonzero, for any sufficiently small $\epsilon$, there is a point $\mathbf{x}_{0}$ between $D_{1}$ and $D_{2}$ such that

$$
\frac{1}{2 C_{* *}}\left|a_{1}\right|\left(\frac{r_{1} r_{2}}{r_{1}+r_{2}}\right) \frac{1}{\epsilon} \leq\left|\nabla u\left(\mathbf{x}_{0}\right)\right| .
$$

The lower bound above is optimal in the sense that there is a positive constant $C^{* *}$ independent of $\epsilon, r_{1}, r_{2}$ and $\left(a_{1}, a_{2}, \cdots, a_{n}\right)$, satisfying that

$$
\|\nabla u\|_{L^{\infty}\left(\mathbb{R}^{3} \backslash\left(D_{1} \cup D_{2}\right)\right)} \leq C^{* *}\left|a_{1}\right|\left(\frac{r_{1} r_{2}}{r_{1}+r_{2}}\right) \frac{1}{\epsilon},
$$

for sufficiently small $\epsilon>0$.

(b) In the case that $a_{1}$ is zero, the gradient of $u$ does not blow up even when the distance $\epsilon$ goes to zero, i.e., there is a positive constant $C_{0}^{* *}$ independent of $\epsilon, r_{1}, r_{2}$ and $\left(a_{1}, a_{2}, \cdots, a_{n}\right)$, satisfying

$$
\|\nabla u\|_{L^{\infty}\left(\mathbb{R}^{3} \backslash\left(D_{1} \cup D_{2}\right)\right)} \leq C_{0}^{* *} \sum_{i=2}^{n}\left|a_{i}\right|,
$$

for sufficiently small $\epsilon>0$.

Similarly to Remark 2.2, the constant $C_{0}^{* *}$ above depends on $r_{1}$ and $r_{2}, \mathrm{i}, \mathrm{e}$, there is a constant $C$ so that

$$
\|\nabla u\|_{L^{\infty}\left(\mathbb{R}^{n} \backslash\left(D_{1} \cup D_{2}\right)\right)} \leq C \max \left\{\frac{r_{1}}{r_{2}}, \frac{r_{2}}{r_{1}}\right\} \sum_{i=2}^{n}\left|a_{i}\right|,
$$

when $a_{1}=0$. The derivation of the inequality above is also presented in the proof of Theorem 2.3 .

In this work, the blow-up estimates in terms of radii is presented only for three or higher dimensional case. Speaking of two dimensions, Ammari et al [2] already provided the optimal bound (5) and (10) in terms of radii of circular inclusions as

$$
\max |\nabla(u-H)| \leq C \sqrt{\frac{r_{1} r_{2}}{r_{1}+r_{2}}} \frac{1}{\sqrt{\epsilon}}
$$

where $r_{1}$ and $r_{2}$ are the radii of circular inclusions. This is also derived in Proposition 3.2 of this paper. As has been mentioned before, the method in this paper is much simpler method. 
Theorem 2.4 (General entire harmonic function $H$ ) Let $D_{1}$ and $D_{2}$ be a pair of balls as assumed in the previous theorems in $n$ dimensions $(n \geq 3)$. We choose a large bounded domain $\Omega$ containing $D_{1}$ and $D_{2}$ for any small $\epsilon>0$. For the sake of convenience, we select the ball $B_{4\left(r_{1}+r_{2}\right)}(0, \cdots, 0)$ as $\Omega$. For any given entire harmonic function $H$, let $u$ be the solution to (1) for $H$.

In three dimensions $(n=3)$, there is a constant $C^{*}$, independent of $\epsilon, r_{1}, r_{2}$ and $\left(a_{1}, a_{2}, a_{3}\right)$, satisfying

$$
\|\nabla(u-H)\|_{L^{\infty}\left(\mathbb{R}^{3} \backslash\left(D_{1} \cup D_{2}\right)\right)} \leq C^{*}\|\nabla H\|_{L^{\infty}(\Omega)}\left(\frac{r_{1} r_{2}}{r_{1}+r_{2}}\right) \frac{1}{|\epsilon \log \epsilon|}
$$

and

$$
\|\nabla u\|_{L^{\infty}\left(\Omega \backslash\left(D_{1} \cup D_{2}\right)\right)} \leq C^{*}\|\nabla H\|_{L^{\infty}(\Omega)}\left(\frac{r_{1} r_{2}}{r_{1}+r_{2}}\right) \frac{1}{|\epsilon \log \epsilon|}
$$

for sufficiently small $\epsilon>0$.

In higher dimensions $(n \geq 4)$, there is a constant $C^{* *}$, independent of $\epsilon, r_{1}, r_{2}$ and $\left(a_{1}, a_{2}, \cdots, a_{n}\right)$, satisfying

$$
\|\nabla(u-H)\|_{L^{\infty}\left(\mathbb{R}^{3} \backslash\left(D_{1} \cup D_{2}\right)\right)} \leq C^{* *}\|\nabla H\|_{L^{\infty}(\Omega)}\left(\frac{r_{1} r_{2}}{r_{1}+r_{2}}\right) \frac{1}{\epsilon}
$$

and

$$
\|\nabla u\|_{L^{\infty}\left(\Omega \backslash\left(D_{1} \cup D_{2}\right)\right)} \leq C^{* *}\|\nabla H\|_{L^{\infty}(\Omega)}\left(\frac{r_{1} r_{2}}{r_{1}+r_{2}}\right) \frac{1}{\epsilon}
$$

for sufficiently small $\epsilon>0$.

\section{Representation of the potential difference}

We introduce a harmonic function $h$ as follows:

$$
\begin{cases}\Delta h=0, & \text { in } \mathbb{R}^{n} \backslash \overline{\left(D_{1} \cup D_{2}\right)}, \\ h=O\left(|\mathbf{x}|^{1-n}\right), & \text { as }|\mathbf{x}| \rightarrow \infty, \\ \left.h\right|_{\partial D_{i}}=k_{i} \text { (constant), } & \\ \int_{\partial D_{i}} \partial_{\nu} h d S=(-1)^{i+1}, & \text { for } i=1,2 .\end{cases}
$$

It is essential in this work to construct the function $h$ because of the following lemma.

Lemma 3.1 ([11]) For a solution $u$ to (11), we have that

$$
\left.u\right|_{\partial D_{1}}-\left.u\right|_{\partial D_{2}}=\int_{\partial D_{1}}\left(\partial_{\nu} h\right) H d S+\int_{\partial D_{2}}\left(\partial_{\nu} h\right) H d S .
$$

Proof. With the boundary condition of $u$ on $\partial D_{1}$ and $\partial D_{2}$, Green's identity for $H$ inside $D_{1}$ and $D_{2}$ yields

$$
\int_{\partial D_{1}} \partial_{\nu}(u-H) d S=\int_{\partial D_{2}} \partial_{\nu}(u-H) d S=0,
$$

and thus

$$
I:=\int_{\partial D_{1}} h \partial_{\nu}(u-H) d S+\int_{\partial D_{2}} h \partial_{\nu}(u-H) d S=0 .
$$


Applying again Green's identity outside $\overline{D_{1} \cup D_{2}}$, we have

$$
0=I=\left(\left.u\right|_{\partial D_{1}}-\left.u\right|_{\partial D_{2}}\right)-\int_{\partial D_{1}}\left(\partial_{\nu} h\right) H d S-\int_{\partial D_{2}}\left(\partial_{\nu} h\right) H d S .
$$

We remark that the above representation (7) is observed by Yun 11] for the purpose of estimating the stresses between two arbitrary shaped inclusions in $\mathbb{R}^{2}$. By constructing a harmonic function $h$ and calculating the right hand side of (7), Yun estimated the potential difference between two adjacent conductors.

The idea to establish $h$ is from the basic theory in electrodynamics, and we use several times the following property of Apollonius circles.

\subsection{Apollonius Circle in $\mathbb{R}^{n}$}

For a ball $B_{r}(\mathbf{c})$ in $\mathbb{R}^{n}$ and a point $\mathbf{p},|\mathbf{p}-\mathbf{c}|>r$, we have

$$
\frac{r}{|\mathbf{p}-\mathbf{c}|} \frac{1}{|\mathbf{x}-R(\mathbf{p})|}=\frac{1}{|\mathbf{x}-\mathbf{p}|}, \quad \text { for all } \mathbf{x} \in \partial B_{r}(\mathbf{c}),
$$

where $R$ is the reflection with respect to $B_{r}(\mathbf{c})$, i.e.,

$$
R(\mathbf{p})=\frac{r^{2}(\mathbf{p}-\mathbf{c})}{|\mathbf{p}-\mathbf{c}|^{2}}+\mathbf{c} .
$$

A simple application of Apollonius circle is estimating the potential difference of the solution to (1) for two circles with different radii.

\subsection{Estimates in $\mathbb{R}^{2}$}

We let

$$
D_{1}=B_{r_{1}}\left(\mathbf{c}_{1}\right) \text { and } D_{2}=B_{r_{2}}\left(\mathbf{c}_{2}\right),
$$

where $\mathbf{c}_{1}=\left(r_{1}+\epsilon, 0\right)$ and $\mathbf{c}_{2}=\left(-r_{2}-\epsilon, 0\right)$, and $R_{i}$ be the reflection with respect to $D_{i}$, in other words,

$$
R_{i}(\mathbf{x})=\frac{r_{i}^{2}\left(\mathbf{x}-\mathbf{c}_{i}\right)}{\left|\mathbf{x}-\mathbf{c}_{i}\right|^{2}}+\mathbf{c}_{i}, i=1,2 .
$$

Let $\mathbf{p}_{1} \in D_{1}$ be the fixed point of $R_{1} \circ R_{2}$, then $R_{2}\left(\mathbf{p}_{1}\right)\left(=: \mathbf{p}_{2}\right)$ is the fixed point of $R_{2} \circ R_{1}$ and $R_{1}\left(\mathbf{p}_{2}\right)=\mathbf{p}_{1}$. From (8),

$$
\frac{\left|\mathbf{x}-\mathbf{p}_{1}\right|}{\left|\mathbf{x}-\mathbf{p}_{2}\right|}= \begin{cases}\frac{r_{1}}{\left|\mathbf{p}_{2}-\mathbf{c}_{1}\right|}, & \text { for } \mathbf{x} \in \partial D_{1}, \\ \frac{\left|\mathbf{p}_{1}-\mathbf{c}_{2}\right|}{r_{2}}, & \text { for } \mathbf{x} \in \partial D_{2} .\end{cases}
$$

Hence, the solution to (6) is

$$
h:=\frac{1}{2 \pi}\left(\log \left|\mathbf{x}-\mathbf{p}_{1}\right|-\log \left|\mathbf{x}-\mathbf{p}_{2}\right|\right)=\frac{1}{2 \pi} \log \left(\frac{\left|\mathbf{x}-\mathbf{p}_{1}\right|}{\left|\mathbf{x}-\mathbf{p}_{2}\right|}\right),
$$

and, from (7), we have the following proposition. 
Proposition 3.2 Let $H\left(x_{1}, x_{2}\right)$ be an entire harmonic function. The solution $u$ to (1) satisfies

$$
\begin{aligned}
\left.u\right|_{\partial D_{1}}-\left.u\right|_{\partial D_{2}} & =H\left(\boldsymbol{p}_{1}\right)-H\left(-\boldsymbol{p}_{2}\right) \\
& =4 \partial_{x_{1}} H(0,0) \sqrt{\frac{r_{1} r_{2}}{r_{1}+r_{2}}} \sqrt{\epsilon}+O(\epsilon) .
\end{aligned}
$$

Referring to the mean value theorem, there exists a point $\boldsymbol{x}_{2}$ between $\partial D_{1}$ and $\partial D_{2}$ such that

$$
\left|\nabla u\left(\boldsymbol{x}_{2}\right)\right| \geq\left|\partial_{x_{1}} H(0,0)\right| \sqrt{\frac{r_{1} r_{2}}{r_{1}+r_{2}}} \frac{1}{\sqrt{\epsilon}} .
$$

for any sufficiently small $\epsilon>0$. Moreover, there is a constat $C$ independent of $\epsilon, r_{1}$ and $r_{2}$ such that

$$
\|\nabla u\|_{L^{\infty}\left(\Omega \backslash\left(D_{1} \cup D_{2}\right)\right)} \leq C\|\nabla H\|_{L^{\infty}(\Omega)} \sqrt{\frac{r_{1} r_{2}}{r_{1}+r_{2}}} \frac{1}{\sqrt{\epsilon}}
$$

where $\Omega=B_{4\left(r_{1}+r_{2}\right)}(0,0)$.

Proof. The fixed points $\mathbf{p}_{i}$ satisfies

$$
\mathbf{p}_{1}=\left(2 \sqrt{\frac{r_{1} r_{2}}{r_{1}+r_{2}}} \sqrt{\epsilon}+O(\epsilon), 0\right) \text { and } \mathbf{p}_{2}=\left(-2 \sqrt{\frac{r_{1} r_{2}}{r_{1}+r_{2}}} \sqrt{\epsilon}+O(\epsilon), 0\right) .
$$

Therefore, we obtain (9) and (10). By virtue of the argument presented by Bao et al. in [4, the upper bound of the gradient is derived from (9). In this paper, the same process as this proposition to derive the upper bound of the gradient is also presented in the proof of Theorem 2.1. Therefore, please refer to the derivation of the upper bound of the gradient in the proof of Theorem 2.4 .

We remark that the same gradient estimate has been obtained by Ammari et al. in 2. They represented $u$ by single layer potentials of the Laplacian with potential functions defined using Kelvin transform $R_{i}, i=1,2$, and obtained the blow-up rate by investigating the potential functions. The novelty of their work is that their estimates is not only for a extreme conductivity but also for a finite positive constant. However, as for the extreme case, our result provides a much simpler method for obtaining the blow-up rate.

\section{Derivation for Theorem 2.1}

Differently from the two dimensional space where the point charge potential, the logarithm, separate the multiplication with ratio $\rho$ into a sum, we cannot constructed $h$ just with two point charge potential functions in higher dimensional space. Therefore, we introduce a sequential process to build $h$.

\subsection{Construct $h$ in $\mathbb{R}^{n}, n \geq 3$}

Let

$$
D_{1}=B_{r_{1}}\left(\mathbf{c}_{1}\right) \text { and } D_{2}=B_{r_{2}}\left(\mathbf{c}_{2}\right),
$$

where

$$
\mathbf{c}_{1}=\left(r_{1}+\epsilon, 0, \ldots, 0\right) \text { and } \mathbf{c}_{2}=\left(-r_{2}-\epsilon, 0, \ldots, 0\right) \text {. }
$$


We start from a harmonic function $h_{1,0}$, defined outside of $\bar{D}_{1}$, which is

$$
h_{1,0}(\mathbf{x})=\frac{1}{\left|\mathbf{x}-\mathbf{c}_{1}\right|^{n-2}} .
$$

Note that $h_{1,0}$ is constant on $\partial D_{1}$. However, it is not constant on $\partial D_{2}$, and we neutralize it by adding auxiliary point charge potential $h_{1,1}$ to make $\left(h_{1,0}+h_{1,1}\right)$ constantly zero on $\partial D_{2}$. From (8), $h_{1,1}$ is defined as

$$
h_{1,1}(\mathbf{x})=\left(\frac{r_{2}}{\left|\mathbf{c}_{2}-\mathbf{c}_{1}\right|}\right)^{n-2} \frac{-1}{\left|\mathbf{x}-R_{2}\left(\mathbf{c}_{1}\right)\right|^{n-2}},
$$

where $R_{i}, i=1,2$, be the reflection with respect to $D_{i}$. On the next step, we add $h_{1,2}$ to $\left(h_{1,0}+h_{1,1}\right)$ and make $\left(h_{1,0}+h_{1,1}+h_{1,2}\right)$ be constant on $\partial D_{1}$, i.e.,

$$
h_{1,2}=\left(\frac{r_{2}}{\left|\mathbf{c}_{2}-\mathbf{c}_{1}\right|}\right)^{n-2}\left(\frac{r_{1}}{\left|\mathbf{c}_{1}-R_{2}\left(\mathbf{c}_{1}\right)\right|}\right)^{n-2} \frac{1}{\left|\mathbf{x}-R_{1}\left(R_{2}\left(\mathbf{c}_{1}\right)\right)\right|^{n-2}}
$$

Consequently, we construct $h_{1, m}, m \in \mathbb{N}$ as

$$
h_{1, m}=\left(q_{1, m}\right)^{n-2} \frac{(-1)^{m}}{\left|\mathbf{x}-\mathbf{c}_{1, m}\right|^{n-2}},
$$

where

$$
\begin{gathered}
\mathbf{c}_{1, m}= \begin{cases}\left(R_{1} R_{2}\right)^{k}\left(\mathbf{c}_{1}\right), & \text { if } m=2 k, k \geq 0, \\
R_{2}\left(R_{1} R_{2}\right)^{k}\left(\mathbf{c}_{1}\right), & \text { if } m=2 k+1, k \geq 0,\end{cases} \\
q_{1, m}=\prod_{j=0}^{m} \rho_{1, j}, \\
\text { for } m \in \mathbb{N},
\end{gathered}
$$

and

$$
\rho_{1, j}= \begin{cases}1, & \text { if } j=0, \\ \frac{r_{1}}{\left|\mathbf{c}_{1}-\mathbf{c}_{1,2 k-1}\right|}, & \text { if } j=2 k, k \geq 1 \\ \frac{r_{2}}{\left|\mathbf{c}_{2}-\mathbf{c}_{1,2 k}\right|}, & \text { if } j=2 k+1 k \geq 0 .\end{cases}
$$

Similarly, we define $h_{2, m}, m \in \mathbb{N}$ as

$$
h_{2, m}=\left(q_{2, m}\right)^{n-2} \frac{(-1)^{m}}{\left|\mathbf{x}-\mathbf{c}_{2, m}\right|^{n-2}},
$$

where

$$
\begin{gathered}
\mathbf{c}_{2, m}= \begin{cases}\left(R_{2} R_{1}\right)^{k}\left(\mathbf{c}_{2}\right), & \text { if } m=2 k, k \geq 0, \\
R_{1}\left(R_{2} R_{1}\right)^{k}\left(\mathbf{c}_{2}\right), & \text { if } m=2 k+1, k \geq 0,\end{cases} \\
q_{2, m}=\prod_{j=0}^{m} \rho_{2, j}, \\
\text { for } m \in \mathbb{N},
\end{gathered}
$$


and

$$
\rho_{2, j}= \begin{cases}1, \quad \text { if } j=0, \\ \frac{r_{2}}{\left|\mathbf{c}_{2}-\mathbf{c}_{2,2 k-1}\right|}, & \text { if } j=2 k, k \geq 1 \\ \frac{r_{1}}{\left|\mathbf{c}_{1}-\mathbf{c}_{2,2 k}\right|}, & \text { if } j=2 k+1 k \geq 0 .\end{cases}
$$

Since $\left(R_{1} R_{2}\right)^{k}\left(\mathbf{c}_{1}\right) \in D_{1}$ and $R_{2}\left(R_{1} R_{2}\right)^{k}\left(\mathbf{c}_{1}\right) \in D_{2}$, we have, for $j \geq 1$,

$$
\rho_{1, j} \leq \max _{i=1,2} \frac{r_{i}}{r_{i}+2 \epsilon}=\frac{1}{1+\frac{2 \epsilon}{r_{\max }}}, \text { where } r_{\max }=\max \left(r_{1}, r_{2}\right) .
$$

By the same way, $\rho_{2, j} \leq \frac{1}{1+\frac{2 \epsilon}{r_{\max }}}$. Hence,

$$
\sum_{m=0}^{\infty}\left(q_{s, m}\right)^{n-2}<\infty, \quad s=1,2
$$

and the two series $\sum_{m=0}^{\infty} h_{i, m}, i=1,2$, are well defined. To get $h$ satisfying the decaying condition, we sum the series $h_{1, m}$ and $h_{2, m}$ with different weights as the following lemma.

Lemma 4.1 The solution to (6) is given by

$$
h(\mathbf{x})=\frac{1}{(2-n) \omega_{n}} \frac{1}{M}\left[Q_{2} \sum_{m=0}^{\infty} \frac{(-1)^{m}\left(q_{1, m}\right)^{n-2}}{\left|\mathbf{x}-\mathbf{c}_{1, m}\right|^{n-2}}-Q_{1} \sum_{m=0}^{\infty} \frac{(-1)^{m}\left(q_{2, m}\right)^{n-2}}{\left|\mathbf{x}-\mathbf{c}_{2, m}\right|^{n-2}}\right],
$$

where $\omega_{n}$ is the area of the unit sphere, and

$$
\begin{gathered}
Q_{s}=\sum_{m=0}^{\infty}\left[(-1)^{m}\left(q_{s, m}\right)^{n-2}\right], \quad s=1,2, \\
M=Q_{2} \sum_{k=0}^{\infty}\left(q_{1,2 k}\right)^{n-2}+Q_{1} \sum_{k=0}^{\infty}\left(q_{2,2 k+1}\right)^{n-2} .
\end{gathered}
$$

Here, $q_{s, m}$ 's and $\mathbf{c}_{s, m}$ 's are defined by (12), (19), (16), (17).

Proof. Let

$$
h_{*}(\mathbf{x})=Q_{2} \sum_{m=0}^{\infty} \frac{(-1)^{m}\left(q_{1, m}\right)^{n-2}}{\left|\mathbf{x}-\mathbf{c}_{1, m}\right|^{n-2}}-Q_{1} \sum_{m=0}^{\infty} \frac{(-1)^{m}\left(q_{2, m}\right)^{n-2}}{\left|\mathbf{x}-\mathbf{c}_{2, m}\right|^{n-2}},
$$

then $h_{*}$ satisfies (6) except the last condition, boundary integral conditions.

Note that

$$
\frac{1}{(2-n) \omega_{n}} \int_{\partial D_{i}} \frac{\partial}{\partial \nu} \frac{1}{|\mathbf{x}-\mathbf{p}|^{n-2}} d \sigma(\mathbf{x})= \begin{cases}0, & \text { for } \mathbf{p} \in \mathbb{R}^{n} \backslash \bar{D}_{i}, \\ 1, & \text { for } \mathbf{p} \in D_{i},\end{cases}
$$


and we have

$$
\begin{aligned}
& \frac{1}{(2-n) \omega_{n}} \int_{\partial D_{1}} \partial_{\nu} h_{*}(\mathbf{x}) d \sigma(\mathbf{x}) \\
& =Q_{2} \sum_{k=0}^{\infty}\left(q_{1,2 k}\right)^{n-2}+Q_{1} \sum_{k=0}^{\infty}\left(q_{2,2 k+1}\right)^{n-2} \\
& =\sum_{k=0}^{\infty}\left(q_{1,2 k}\right)^{n-2} \sum_{k=0}^{\infty}\left(q_{2,2 k}\right)^{n-2}-\sum_{k=0}^{\infty}\left(q_{1,2 k+1}\right)^{n-2} \sum_{k=0}^{\infty}\left(q_{2,2 k+1}\right)^{n-2} \\
& =Q_{1} \sum_{k=0}^{\infty}\left(q_{2,2 k}\right)^{n-2}+Q_{2} \sum_{k=1}^{\infty}\left(q_{1,2 k+1}\right)^{n-2} \\
& =-\frac{1}{(2-n) \omega_{n}} \int_{\partial D_{2}} \partial_{\nu} h_{*}(\mathbf{x}) d \sigma(\mathbf{x}) .
\end{aligned}
$$

Lemma 4.2 Assume that the dimension $n$ is 3 and the distance $\epsilon$ is sufficiently small. Then, there is a positive constant $C$ independent of $r_{1}, r_{2}$, and $\epsilon$ satisfying the following properties:

- Estimates for $\sum_{m=0}^{\infty} q_{s, m}$ :

$$
\begin{aligned}
& \frac{1}{C} \frac{d}{d+1}|\log \epsilon| \leq \sum_{m=0}^{\infty} q_{1, m} \leq C \frac{d}{d+1}|\log \epsilon|, \\
& \frac{1}{C} \frac{1}{d+1}|\log \epsilon| \leq \sum_{m=0}^{\infty} q_{2, m} \leq C \frac{1}{d+1}|\log \epsilon|
\end{aligned}
$$

where $d=\frac{r_{2}}{r_{1}}$.

- Estimates for $Q_{s}$ :

$$
\begin{aligned}
& \frac{1}{C} \frac{1}{d+1} \leq Q_{1} \leq C \frac{1}{d+1}, \\
& \frac{1}{C} \frac{d}{d+1} \leq Q_{2} \leq C \frac{d}{d+1}
\end{aligned}
$$

where $Q_{1}$ and $Q_{2}$ are defined in Lemma 4.1.

- Estimates for $\sum_{k=0}^{\infty}\left(c_{s, 2 k}\right)\left(q_{s, 2 k}\right)-\sum_{k=0}^{\infty}\left(c_{s, 2 k+1}\right)\left(q_{s, 2 k+1}\right)$ :

$$
\frac{r_{s}}{C} \leq(-1)^{s+1}\left[\sum_{k=0}^{\infty}\left(c_{s, 2 k}\right)\left(q_{s, 2 k}\right)-\sum_{k=0}^{\infty}\left(c_{s, 2 k+1}\right)\left(q_{s, 2 k+1}\right)\right] \leq C r_{s} .
$$

Now, we are ready to prove Theorem 2.1 


\subsection{Proof of Theorem 2.1}

\section{Potential Difference}

We first consider the case of $H\left(x_{1}, x_{2}, x_{3}\right)=x_{1}$. Then, Lemma 4.1 implies

$$
\begin{aligned}
\left.u\right|_{\partial D_{1}}-\left.u\right|_{\partial D_{2}}= & \frac{Q_{2}}{M} \sum_{k=0}^{\infty}\left(c_{1,2 k}\right)\left(q_{1,2 k}\right)+\frac{Q_{1}}{M} \sum_{k=0}^{\infty}\left(c_{2,2 k+1}\right)\left(q_{2,2 k+1}\right) \\
& -\frac{Q_{2}}{M} \sum_{k=0}^{\infty}\left(c_{1,2 k+1}\right)\left(q_{1,2 k+1}\right)-\frac{Q_{1}}{M} \sum_{k=0}^{\infty}\left(c_{2,2 k}\right)\left(q_{2,2 k}\right),
\end{aligned}
$$

where $c_{s, j}$ is the $x_{1}$-coordinate of $\mathbf{c}_{s, j}$ for $s=1,2, j \in \mathbb{N}$, i.e.,

$$
\left(c_{s, j}, 0,0\right)=\mathbf{c}_{s, j} .
$$

Lemma 4.2 allows one to estimate four positive valued terms in the right hand side of (23) so that

$$
\begin{aligned}
M & =Q_{2} \sum_{k=0}^{\infty} q_{1,2 k}+Q_{1} \sum_{k=0}^{\infty} q_{2,2 k+1} \\
& \simeq\left[\frac{d^{2}}{(d+1)^{2}}+\frac{1}{(d+1)^{2}}\right]|\log \epsilon| \\
& \simeq|\log \epsilon| .
\end{aligned}
$$

In total, we obtain

$$
|u|_{\partial D_{1}}-\left.u\right|_{\partial D_{2}} \mid \simeq \frac{r_{1} r_{2}}{r_{1}+r_{2}} \frac{1}{|\log \epsilon|} .
$$

In the case of $H=a_{2} x_{2}+a_{3} x_{3}$, the integration (17) is zero; all point charges of $h$ lie on $x_{1}$ axis. Therefore, there is no potential difference between inclusions, and we have established the estimate for the potential difference between $D_{1}$ and $D_{2}$.

Therefore, for $H=\sum_{i=1}^{3} a_{i} x_{i}$,

$$
|u|_{\partial D_{1}}-\left.u\right|_{\partial D_{2}}|\simeq| a_{1} \mid \frac{r_{1} r_{2}}{r_{1}+r_{2}} \frac{1}{|\log \epsilon|}
$$

\section{Lower bound}

The lower bound is obtained by simply applying the mean value theorem. Since $|u|_{D_{1}}-\left.u\right|_{D_{2}} \mid$ behaves as $1 /|\log \epsilon|$, the gradient behaves as $1 /(\epsilon|\log \epsilon|)$, and, more precisely, there is a point $\mathbf{x}_{0}$ between $D_{1}$ and $D_{2}$ satisfying that

$$
\frac{1}{C}\left|a_{1}\right|\left(\frac{r_{1} r_{2}}{r_{1}+r_{2}}\right) \frac{1}{|\epsilon \log \epsilon|} \leq\left|\nabla u\left(\mathbf{x}_{0}\right)\right|
$$

where $H=\sum_{i=1}^{3} a_{i} x_{i}$.

\section{Upper bound}

The upper bound of the gradient is derived by applying the methods presented by Bao et al 4 . 
We assume that $r_{1} \geq r_{2}$, and let

$$
\frac{1}{r_{1}} D_{i}=\left\{\mathbf{x} \in \mathbb{R}^{3} \mid r_{1} \mathbf{x} \in D_{i}\right\}, \quad i=1,2 .
$$

Note that $\frac{1}{r_{1}} D_{1}$ is a unit sphere. Define a bounded domain $\Omega$, containing $\frac{1}{r_{1}} D_{1}$ and $\frac{1}{r_{1}} D_{2}$ independently of $\epsilon$, as the sphere $B_{4}(0,0,0)$. Now, for a solution $u$ to (11) for $H(\mathbf{x})=$ $\sum_{i=1}^{3} a_{i} x_{i}$, define the scaled function $\tilde{u}$ as

$$
\widetilde{u}(\mathbf{x}):=\frac{1}{r_{1}} u\left(r_{1} \mathbf{x}\right) .
$$

Then $\widetilde{u}$ is also the solution to (11) for $H(\mathbf{x})=\sum_{i=1}^{3} a_{i} x_{i}$ with $\frac{1}{r_{1}} D_{1}$ and $\frac{1}{r_{1}} D_{2}$ instead of $D_{1}$ and $D_{2}$. The estimate for the difference of $u$ between $\partial D_{1}$ and $\partial D_{2}$ in this theorem yields

$$
|\widetilde{u}|_{\partial\left(\frac{1}{r_{1}} D_{1}\right)}-\left.\widetilde{u}\right|_{\partial\left(\frac{1}{r_{1}} D_{2}\right)}|\simeq| a_{1} \mid \frac{d}{1+d} \frac{1}{|\log \delta|},
$$

where

$$
d=\frac{r_{2}}{r_{1}} \text { and } \delta=\frac{\epsilon}{r_{1}} .
$$

By the maximum principle, we have

$$
\begin{aligned}
\|\widetilde{u}-H\|_{L^{\infty}(\partial \Omega)} & \leq|\widetilde{u}|_{\partial \frac{1}{r_{1} D_{1}}}-\left.\widetilde{u}\right|_{\partial \frac{1}{r_{1}} D_{2}} \mid+2\|H\|_{L^{\infty}(\Omega)} \\
& \leq C\left(\left|a_{1}\right|\left(\frac{d}{1+d}\right) \frac{1}{|\log \delta|}+\left|a_{1}\right|+\left|a_{2}\right|+\left|a_{3}\right|\right),
\end{aligned}
$$

and, as a result,

$$
\|\widetilde{u}\|_{L^{\infty}(\partial \Omega)} \leq C^{\prime}\left(\left|a_{1}\right|\left(\frac{d}{1+d}\right) \frac{1}{|\log \delta|}+\left|a_{1}\right|+\left|a_{2}\right|+\left|a_{3}\right|\right) .
$$

To estimate $|\nabla \tilde{u}|$ on $\partial\left(\frac{1}{r_{1}} D_{1} \cup \frac{1}{r_{1}} D_{2}\right)$, we define $v_{3}$ as in [4]:

$$
\begin{cases}\Delta v_{3}=0 & \text { in } \Omega \backslash \overline{\left(\frac{1}{r_{1}} D_{1} \cup \frac{1}{r_{1}} D_{2}\right)} \\ v_{3}=0 & \text { on } \partial\left(\frac{1}{r_{1}} D_{1} \cup \frac{1}{r_{1}} D_{2}\right) \\ v_{3}=-\widetilde{u} & \text { on } \partial \Omega\end{cases}
$$

We draw the attention of readers to Lemma 4.34 .4 which are modified from 4 to fit our problem. For a reader's convenient, we provide the proofs at the end of this section.

Lemma 4.3 (4] There is a constant $C$ independent of $d$ and $\epsilon$ such that

$$
\left\|\nabla\left(\widetilde{u}+v_{3}\right)\right\|_{L^{\infty}\left(\Omega \backslash\left(\frac{1}{r_{1}} D_{1} \cup \frac{1}{r_{1}} D_{2}\right)\right)} \leq C\left|a_{1}\right|\left(\frac{d}{1+d}\right) \frac{1}{|\delta \log \delta|} .
$$

Now, in estimating $|\nabla \tilde{u}|$ on $\partial\left(\frac{1}{r_{1}} D_{1} \cup \frac{1}{r_{1}} D_{2}\right)$, it is remained to be derived an upper bound of $\left|\nabla v_{3}\right|$ on $\partial\left(\frac{1}{r_{1}} D_{1} \cup \frac{1}{r_{1}} D_{2}\right)$. To do that, we define the harmonic function $\rho$ in $\Omega \backslash\left(\frac{1}{r_{1}} D_{1} \cup \frac{1}{r_{1}} D_{2}\right)$ as in $[4$ :

$$
\begin{cases}\triangle \rho=0 & \text { in } \Omega \backslash\left(\frac{1}{r_{1}} D_{1} \cup \frac{1}{r_{1}} D_{2}\right), \\ \rho=0 & \text { on } \partial\left(\frac{1}{r_{1}} D_{1} \cup \frac{1}{r_{1}} D_{2}\right), \\ \rho=1 & \text { on } \partial \Omega\end{cases}
$$


Note that $v_{3}= \pm\|\widetilde{u}\|_{L^{\infty}(\partial \Omega)} \rho=0$ on $\partial\left(\frac{1}{r_{1}} D_{1} \cup \frac{1}{r_{1}} D_{2}\right)$. Moreover, from the fact that $v_{3}=-\widetilde{u}$ on the $\partial \Omega$ and the maximum principle, for $x \in \Omega \backslash\left(\frac{1}{r_{1}} D_{1} \cup \frac{1}{r_{1}} D_{2}\right)$, we have $-\|\widetilde{u}\|_{L^{\infty}(\partial \Omega)} \rho \leq v_{3} \leq\|\widetilde{u}\|_{L^{\infty}(\partial \Omega)} \rho$. Therefore, by Hopf's Lemma and the maximum principle,

$$
\left\|\nabla v_{3}\right\|_{L^{\infty}\left(\Omega \backslash\left(\frac{1}{r_{1}} D_{1} \cup \frac{1}{r_{1}} D_{2}\right)\right)} \leq\|\widetilde{u}\|_{L^{\infty}(\partial \Omega)}\|\nabla \rho\|_{L^{\infty}\left(\Omega \backslash\left(\frac{1}{r_{1}} D_{1} \cup \frac{1}{r_{1}} D_{2}\right)\right)} .
$$

We apply the following lemma to calculate $\left\|\nabla v_{3}\right\|_{L^{\infty}\left(\Omega \backslash\left(\frac{1}{r_{1}} D_{1} \cup \frac{1}{r_{1}} D_{2}\right)\right)}$.

Lemma 4.4 (4]) There is a constant $C$ such that

$$
\|\nabla \rho\|_{L^{\infty}\left(\partial \frac{1}{r_{1}} D_{1} \cup \partial \frac{1}{r_{1}} D_{2}\right)} \leq C \frac{1}{d}
$$

for $\epsilon$ small enough.

Applying Lemma 4.4, we have

$$
\left\|\nabla v_{3}\right\|_{L^{\infty}\left(\partial\left(\frac{1}{r_{1}} D_{1} \cup \frac{1}{r_{1}} D_{2}\right)\right)} \leq C \frac{1}{d}\left(\left|a_{1}\right|\left(\frac{d}{1+d}\right) \frac{1}{|\log \delta|}+\left|a_{1}\right|+\left|a_{2}\right|+\left|a_{3}\right|\right) .
$$

Two bounds (24) and (25) yield

$$
\begin{aligned}
\|\nabla u\|_{L^{\infty}\left(\partial\left(D_{1} \cup D_{2}\right)\right)} & =\|\nabla \widetilde{u}\|_{L^{\infty}\left(\partial\left(\frac{1}{r_{1}} D_{1} \cup \frac{1}{r_{1}} D_{2}\right)\right)} \\
& \leq C\left(\left|a_{1}\right|\left(\frac{d}{1+d}\right) \frac{1}{|\delta \log \delta|}+\frac{1}{d}\left(\left|a_{2}\right|+\left|a_{3}\right|\right)\right) \\
& \leq C^{\prime}\left(\left|a_{1}\right|\left(\frac{r_{1} r_{2}}{r_{1}+r_{2}}\right) \frac{1}{|\epsilon \log \epsilon|}+\frac{1}{d}\left(\left|a_{2}\right|+\left|a_{3}\right|\right)\right)
\end{aligned}
$$

for sufficiently small $\epsilon>0$. Since $|\nabla H|$ is bounded by $\left|a_{1}\right|+\left|a_{2}\right|+\left|a_{3}\right|$, we have

$$
\|\nabla(u-H)\|_{L^{\infty}\left(\partial\left(D_{1} \cup D_{2}\right)\right)} \leq C\left(\left|a_{1}\right|\left(\frac{r_{1} r_{2}}{r_{1}+r_{2}}\right) \frac{1}{|\epsilon \log \epsilon|}+\frac{1}{d}\left(\left|a_{2}\right|+\left|a_{3}\right|\right)\right) .
$$

By the harmonicity of $u-H$ in $\mathbb{R}^{3} \backslash\left(D_{1} \cup D_{2}\right),\|\nabla(u-H)\|_{L^{\infty}\left(\mathbb{R}^{3} \backslash\left(D_{1} \cup D_{2}\right)\right)}$ has the same upper bound as the above. The fact of $|\nabla H| \leq\left|a_{1}\right|+\left|a_{2}\right|+\left|a_{3}\right|$ is again used so that

$$
\|\nabla u\|_{L^{\infty}\left(\mathbb{R}^{3} \backslash\left(D_{1} \cup D_{2}\right)\right)} \leq C\left(\left|a_{1}\right|\left(\frac{r_{1} r_{2}}{r_{1}+r_{2}}\right) \frac{1}{|\epsilon \log \epsilon|}+\frac{1}{d}\left(\left|a_{2}\right|+\left|a_{3}\right|\right)\right) .
$$

Therefore, we obtain the upper bound of the gradient estimate.

\section{Proof of Lemma 4.3}

From definition, $\widetilde{u}+v_{3}$ is constant on $\partial \frac{1}{r_{1}} D_{1}$ and $\frac{1}{r_{1}} D_{1}$ is a unit ball. By Kelvin transform, $\widetilde{u}+v_{3}$ can be extended harmonically to $\Omega \backslash\left(\frac{1}{r_{1}} D_{1, \delta^{\prime}} \cup \frac{1}{r_{1}} D_{2}\right)$ where

$$
\frac{1}{r_{1}} D_{1, \delta^{\prime}}=\left\{\mathbf{x} \in \frac{1}{r_{1}} D_{1} \mid \operatorname{dist}\left(\mathbf{x}, \partial \frac{1}{r_{1}} D_{1}\right)>\delta^{\prime}\right\}
$$

and

$$
\delta^{\prime}=1-\frac{1}{1+2 \delta} .
$$


Similarly, $\widetilde{u}+v_{3}$ can be also extended harmonically to $\Omega \backslash\left(\frac{1}{r_{1}} D_{1, \delta^{\prime}} \cup \frac{1}{r_{1}} D_{2, \delta^{\prime \prime}}\right)$ where

$$
\frac{1}{r_{1}} D_{2, \delta^{\prime \prime}}=\left\{\mathrm{x} \in \frac{1}{r_{1}} D_{2} \mid \operatorname{dist}\left(\mathrm{x}, \partial \frac{1}{r_{1}} D_{2}\right)>\delta^{\prime \prime}\right\}
$$

and

$$
\delta^{\prime \prime}=d-\frac{d^{2}}{d+2 \delta} .
$$

Furthermore, by the standard estimate for the extension, there is a constant $C$ such that

$$
\max _{\Omega \backslash\left(\frac{1}{r_{1}} D_{1, \delta^{\prime}} \cup \frac{1}{r_{1}} D_{2, \delta^{\prime \prime}}\right)}\left(\widetilde{u}-v_{3}\right)-\min _{\Omega \backslash\left(\frac{1}{r_{1}} D_{1, \delta^{\prime}} \cup \frac{1}{r_{1}} D_{2, \delta^{\prime \prime}}\right)}\left(\widetilde{u}-v_{3}\right) \leq C\left|a_{1}\right|\left(\frac{d}{1+d}\right) \frac{1}{|\log \delta|}
$$

for sufficiently small $\epsilon>0$. Note that

$$
\delta^{\prime} \approx 2 \delta \text { and } \delta^{\prime \prime} \approx 2 \delta \text {. }
$$

By the gradient estimate for harmonic functions, we have

$$
\left\|\nabla\left(\widetilde{u}+v_{3}\right)\right\|_{L^{\infty}\left(\Omega \backslash\left(\frac{1}{r_{1}} D_{1} \cup \frac{1}{r_{1}} D_{2}\right)\right)} \leq C\left|a_{1}\right|\left(\frac{d}{1+d}\right) \frac{1}{|\delta \log \delta|} .
$$

Proof of Lemma 4.4

Let $\rho_{i}(i=1,2)$ be the solution to

$$
\begin{cases}\triangle \rho_{i}=0 & \text { in } \Omega \backslash\left(\frac{1}{r_{1}} D_{i}\right) \\ \rho_{i}=0 & \text { on } \partial\left(\frac{1}{r_{1}} D_{i}\right) \\ \rho_{i}=1 & \text { on } \partial \Omega\end{cases}
$$

Then $\rho=\rho_{i}$ on the $\partial \Omega \cup \partial D_{i}$. The maximum principle yields to $\rho_{i} \leq \rho$. Note that the radii of $\frac{1}{r_{1}} D_{1}$ and $\frac{1}{r_{1}} D_{2}$ are 1 and $d$ respectively.

Consider the harmonic function $v$ which is the solution to

$$
\begin{cases}\Delta v=0 & \text { in } B_{4}(0, \cdots, 0) \backslash B_{r_{0}}\left(\mathbf{c}_{0}\right), \\ v=0 & \text { on } \partial B_{r_{0}}\left(\mathbf{c}_{0}\right), \\ v=1 & \text { on } \partial B_{4}(0, \cdots, 0),\end{cases}
$$

where $r_{0} \leq 1$ and $\left|\mathbf{c}_{0}\right| \leq 2$. Let the harmonic function $w$ be as

$$
w= \begin{cases}\left(\frac{1}{2^{n-2}}-\frac{1}{r_{0}{ }^{n-2}}\right)^{-1}\left(\frac{1}{\left|\mathbf{x}-\mathbf{c}_{0}\right|^{n-2}}-\frac{1}{r_{0}{ }^{n-2}}\right) & \text { for } n \geq 3 \\ \left(\log 2-\log r_{0}\right)\left(\log \left|\mathbf{x}-\mathbf{c}_{0}\right|-\log r_{0}\right) & \text { for } n=2\end{cases}
$$

Then,

$$
w \geq 1=v \text { on } \partial B_{4}(0, \cdots, 0)
$$

and

$$
w=v \text { on } \partial B_{r_{0}}\left(\mathbf{c}_{0}\right) .
$$

Then, there is a constant $C$, independent of $r_{0}$ and $\mathbf{c}_{0}$, satisfying

$$
\|\nabla v\|_{L^{\infty}\left(\partial B_{r_{0}}\left(\mathbf{c}_{0}\right)\right)} \leq C \frac{1}{r_{0}} .
$$


By Hopf's Lemma, we have

$$
-\partial_{\nu} w \geq-\partial_{\nu} v \geq 0 \text { on } \partial B_{r_{0}}\left(\mathbf{c}_{0}\right)
$$

and there is a constant $C$, independent of $\mathbf{c}_{0}$ and $r_{0}$, satisfying

$$
-\partial_{\nu} w \leq C \frac{1}{r_{0}} .
$$

Therefore, we obtain

$$
\|\nabla v\|_{L^{\infty}\left(\partial B_{r_{0}}\left(\mathbf{c}_{0}\right)\right)}=-\partial_{\nu} w \leq C \frac{1}{r_{0}} .
$$

It follows from (26) and Hopf's Lemma that

$$
\|\nabla \rho\|_{L^{\infty}\left(\partial \frac{1}{r_{1}} D_{1}\right)} \leq\left\|\nabla \rho_{1}\right\|_{L^{\infty}\left(\partial \frac{1}{r_{1}} D_{1}\right)} \leq C
$$

and

$$
\|\nabla \rho\|_{L^{\infty}\left(\partial \frac{1}{r_{1}} D_{2}\right)} \leq\left\|\nabla \rho_{2}\right\|_{L^{\infty}\left(\partial \frac{1}{r_{1}} D_{2}\right)} \leq C \frac{1}{d}
$$

\subsection{Proof of Lemma 4.2}

We begin by considering the position sequences $\mathbf{c}_{1, m}$ and $\mathbf{c}_{2, m}$, because the quantities like $q_{i, m}$ and $\rho_{i, j}$ used in the derivation are yielded by these position sequences $\mathbf{c}_{i, m}$. Referring to the relation (12), the successor $\mathbf{c}_{1, m+2}$ to $\mathbf{c}_{1, m}$ is determined by the twice refection $R_{1} \circ R_{2}$. For $\mathbf{x}=(x, 0, \ldots, 0) \in D_{1}$, the twice reflected point $R_{1}\left(R_{2}(\mathbf{x})\right)=\left(x^{\prime}, 0, \ldots, 0\right)$ is given by

$$
x^{\prime}=r_{1}+\epsilon-\frac{r_{1}^{2}}{r_{1}+r_{2}+2 \epsilon-\frac{r_{2}^{2}}{x+r_{2}+\epsilon}} .
$$

For the sake of convenience, we assume that

$$
\delta=\frac{\epsilon}{r_{1}}, d=\frac{r_{2}}{r_{1}}, \text { and } y_{j}=\frac{c_{1, j}}{r_{1}}, \quad j \in \mathbb{N},
$$

where $c_{1, j}$ is the $x_{1}$-coordinate of $\mathbf{c}_{1, j}$. Then, we have the relation

$$
\begin{aligned}
y_{2 k} y_{2 k-2}+\frac{d+(1+3 d) \delta+2 \delta^{2}}{1+d+2 \delta} y_{2 k}-\frac{d+(3+d) \delta+2 \delta^{2}}{1+d+2 \delta} y_{2 k-2} & \\
& -\frac{4 d \delta+3(1+d) \delta^{2}+2 \delta^{3}}{1+d+2 \delta}=0 .
\end{aligned}
$$

Let $\mathbf{p}=(p, 0, \ldots, 0) \in D_{1}$ be the fixed point of $\left(R_{1} \circ R_{2}\right)$, then $\frac{p}{r_{1}}$ is the limit point of $y_{2 k}$ and satisfies

$$
\left(\frac{p}{r_{1}}\right)^{2}+\frac{2(d-1) \delta}{1+d+2 \delta}\left(\frac{p}{r_{1}}\right)-\frac{4 d \delta+3(1+d) \delta^{2}+2 \delta^{3}}{1+d+2 \delta}=0
$$

and, as a results,

$$
\frac{p}{r_{1}}=2 \sqrt{\frac{d}{d+1}} \sqrt{\delta}+O(\delta)
$$


Here, we have a constant $C$ independent of $d$ so that

$$
|O(\sqrt{\delta})| \leq C \sqrt{\delta} \text { for sufficiently small } \delta>0 .
$$

It follows from (28) that

$$
\begin{aligned}
\left(y_{2 k}-\frac{p}{r_{1}}\right)\left(y_{2 k-2}-\frac{p}{r_{1}}\right)+( & \left.\frac{d+(1+3 d) \delta+2 \delta^{2}}{1+d+2 \delta}+\frac{p}{r_{1}}\right)\left(y_{2 k}-\frac{p}{r_{1}}\right) \\
-\left(\frac{d+(3+d) \delta+2 \delta^{2}}{1+d+2 \delta}-\frac{p}{r_{1}}\right)\left(y_{2 k-2}-\frac{p}{r_{1}}\right) & =0 .
\end{aligned}
$$

For simplicity, let

$$
z_{2 k}=y_{2 k}-\frac{p}{r_{1}}
$$

then

$$
1+\left(\frac{d+(1+3 d) \delta+2 \delta^{2}}{1+d+2 \delta}+\frac{p}{r_{1}}\right) \frac{1}{z_{2 k-2}}-\left(\frac{d+(3+d) \delta+2 \delta^{2}}{1+d+2 \delta}-\frac{p}{r_{1}}\right) \frac{1}{z_{2 k}}=0 .
$$

Further, let

$$
A=\frac{\left(\frac{d+(1+3 d) \delta+2 \delta^{2}}{1+d+2 \delta}\right)+\frac{p}{r_{1}}}{\left(\frac{d+(3+d) \delta+2 \delta^{2}}{1+d+2 \delta}\right)-\frac{p}{r_{1}}} \text { and } B=\frac{1}{2\left(\frac{(d-1) \delta}{1+d+2 \delta}+\frac{p}{r_{1}}\right)},
$$

this can be rewritten as

$$
\left(\frac{1}{z_{2 k}}+B\right)=A\left(\frac{1}{z_{2 k-2}}+B\right) .
$$

Therefore, the sequence $y_{2 k}$ is expressed as

$$
\begin{aligned}
y_{2 k} & =z_{2 k}+\frac{p}{r_{1}} \\
& =\frac{1}{\left(\frac{1}{z_{0}}+B\right) A^{k}-B}+\frac{p}{r_{1}} \\
& =\frac{1}{\left(\frac{1}{1+\delta-\frac{p}{r_{1}}}+B\right) A^{k}-B}+\frac{p}{r_{1}} .
\end{aligned}
$$

\subsubsection{Estimates for $y_{j}$}

We simplify (30) under the assumption that $\delta$ is sufficiently small. Our strategy is to choose an appropriate $N$ so that $\sum_{k<N}$ is dominant in the following series calculation, for example in $\sum\left(q_{1,2 m}-q_{1,2 m+1}\right)$, and estimate the series separately for smaller and larger sub-indices.

From now on, we use the big $O$ notation frequently. The equation $f=g+O(\delta)$ means that there exist a constant $C$ independent of $\delta$ such that $|f-g| \leq C \delta$ for small enough $\delta>0$. In this paper, $C$ is assumed additionally to be independent of $d$ and $k$ as well. We define $O(\sqrt{\delta})$ similarly.

The expression (30) of $y_{j}$ is too complicated to well describe the dependency of $y_{j}$ with respect to $d$ and $\delta$. Thus, a simplified expressions is established, provided that the distance $\delta$ is small enough. As for our strategy, we choose an appropriate number $N \simeq \sqrt{\epsilon}$ so that 
the sequence terms of $k \leq N$ are dominant in the sequence $y_{k}$, and thus estimate $y_{2 k}$ in two cases of $k \leq N$ and $k \geq N$ separately.

From the definition of $A$ and $B$, we have

$$
\begin{aligned}
A & =\frac{\frac{d}{1+d}+O(\delta)+\frac{p}{r_{1}}}{\frac{d}{1+d}+O(\delta)-\frac{p}{r_{1}}}=1+2 \frac{d+1}{d} \frac{p}{r_{1}}+\frac{d+1}{d} O(\delta) \\
& =1+4 \sqrt{\frac{d+1}{d}} \sqrt{\delta}+\frac{d+1}{d} O(\delta)
\end{aligned}
$$

and

$$
\begin{aligned}
\sqrt{\delta} B & =\frac{\sqrt{\delta}}{4 \sqrt{\frac{d}{d+1}} \sqrt{\delta}+O(\delta)} \\
& =\frac{1}{4 \sqrt{\frac{d}{d+1}}}+\frac{d+1}{d} O(\sqrt{\delta}) .
\end{aligned}
$$

By a standard argument, one can show that for $x \in(0,2)$ and $(1+x)^{k} \leq 2$,

$$
(1+x)^{k} \leq 1+k x+k^{2} x^{2} .
$$

For $k \leq \frac{\log 2}{8} \sqrt{\frac{d}{d+1}} \frac{1}{\sqrt{\delta}}$, we have $\left(1+8 \sqrt{\frac{d+1}{d}} \sqrt{\delta}\right)^{k} \leq 2$. This yields

$$
\begin{aligned}
A^{k} & =1+k\left(4 \sqrt{\frac{d+1}{d}} \sqrt{\delta}+\frac{d+1}{d} O(\delta)\right)+\frac{d+1}{d} k^{2} O(\delta) \\
& =1+4 k \sqrt{\frac{d+1}{d}} \sqrt{\delta}+\frac{d+1}{d} k^{2} O(\delta) .
\end{aligned}
$$

Hence, the estimate implies

$$
\begin{aligned}
y_{2 k} & =\frac{1}{(B+1+O(\sqrt{\delta}))\left(1+4 k \sqrt{\frac{d+1}{d}} \sqrt{\delta}+\frac{d+1}{d} k^{2} O(\delta)\right)-B}+\frac{p}{r_{1}} \\
& =\frac{1}{1+k \frac{d+1}{d}+\left(\frac{d+1}{d}\right)^{\frac{3}{2}} k^{2} O_{1}(\sqrt{\delta})}+\frac{p}{r_{1}} .
\end{aligned}
$$

Here is a constant $C_{1}>0$ independent of $\delta, d$ and $k$ such that

$$
\left|O_{1}(\sqrt{\delta})\right| \leq C_{1} \sqrt{\delta}
$$

We take the integer $N$ as

$$
N=\min _{n \in \mathbb{N}}\left\{n \geq \frac{1}{C_{1}} \frac{\log 2}{8} \sqrt{\frac{d}{d+1}} \frac{1}{\sqrt{\delta}}\right\} .
$$

Then, for $k \leq N$,

$$
y_{2 k}=\frac{d}{k(d+1)+d}+\sqrt{\frac{d}{d+1}} O(\sqrt{\delta}),
$$


and

$$
\begin{aligned}
y_{2 k+1} & =\frac{1}{r_{1}}\left(-r_{2}-\epsilon+\frac{r_{2}^{2}}{r_{1} y_{2 k}+r_{2}+\epsilon}\right) \\
& =-d-\delta+\frac{d^{2}}{\frac{d}{k(d+1)+d}+d+\sqrt{\frac{d}{d+1}} O(\sqrt{\delta})} \\
& =-\frac{d}{d+1} \frac{1}{k+1}+\sqrt{\frac{d}{d+1}} O(\sqrt{\delta}) .
\end{aligned}
$$

In the case of $k \geq N$, we use the fact that the sequence $y_{1,2 k}$ is decreasing to $\frac{p}{r_{1}}$, i.e., $y_{0}>y_{2}>\cdots>y_{2 k}>y_{2(k+1)}>\cdots>\frac{p}{r_{1}}$. Here (31) yields $y_{1,2 N}=\sqrt{\frac{d}{d+1}} O(\sqrt{\delta})$. From the estimate (29) for $\frac{p}{r_{1}}$, we have

$$
C \sqrt{\frac{d}{d+1}} \sqrt{\delta}>y_{2 k}>\sqrt{\frac{d}{d+1}} \sqrt{\delta}
$$

and

$$
C \sqrt{\frac{d}{d+1}} \sqrt{\delta}>-y_{2 k+1}>\sqrt{\frac{d}{d+1}} \sqrt{\delta} .
$$

Therefore, all estimates (31), (32), (33) and (34) for $y_{2 k}$ and $y_{2 k+1}$ are obtained.

\subsubsection{Estimates for $\sum_{m=0}^{\infty} q_{s, m}$}

We consider the estimate for

$$
q_{1, m}=\prod_{j=0}^{m} \rho_{1, j} .
$$

For $k \leq N$, from (14), (31) and (32), we have

$$
\begin{aligned}
& \rho_{1,2 k+1}=\frac{d}{d+\delta+y_{2 k}}=\frac{k(d+1)+d}{(k+1)(d+1)}\left(1+\frac{1}{\sqrt{d(d+1)}} O(\sqrt{\delta})\right), \\
& \rho_{1,2 k+2}=\frac{1}{1+\delta-y_{2 k+1}}=\frac{(k+1)(d+1)}{(k+1)(d+1)+d}\left(1+\sqrt{\frac{d}{d+1}} O(\sqrt{\delta})\right),
\end{aligned}
$$

and

$$
\left(\rho_{1,2 k+1}\right)\left(\rho_{1,2 k+2}\right)=\frac{k(d+1)+d}{(k+1)(d+1)+d}\left(1+\sqrt{\frac{d+1}{d}} O(\sqrt{\delta})\right) .
$$

In the case of $m \leq N$, they lead to

$$
\begin{aligned}
q_{1,2 m} & =\left(\prod_{k=0}^{m-1} \rho_{1,2 k+1} \rho_{1,2 k+2}\right)=\frac{d}{m(d+1)+d}\left(1+\sqrt{\frac{d+1}{d}} O(\sqrt{\delta})\right)^{m} \\
& =\frac{d}{m(d+1)+d}\left(1+m \sqrt{\frac{d+1}{d}} O(\sqrt{\delta})\right), \\
q_{1,2 m+1} & =\left(q_{1,2 m}\right)\left(\rho_{1,2 m+1}\right) \\
& =\frac{d}{(m+1)(d+1)}\left(1+m \sqrt{\frac{d+1}{d}} O(\sqrt{\delta})\right) .
\end{aligned}
$$


Thus, there exists a positive constant $C$ such that, for $s=0,1$,

$$
\frac{1}{C} \frac{d}{d+1} \frac{1}{m+1} \leq q_{1,2 m+s} \leq C \frac{d}{d+1} \frac{1}{m+1}, \quad 1 \leq m \leq N
$$

and, therefore,

$$
\frac{1}{\tilde{C}} \frac{d}{d+1}|\log \delta| \leq \sum_{m \leq N}\left(q_{1,2 m}+q_{1,2 m+1}\right) \leq \tilde{C} \frac{d}{d+1}|\log \delta| .
$$

In the case of $m \geq N$, (33) and (34) yield, for $s=1,2$,

$$
\frac{1}{C} \frac{1}{1+\sqrt{\frac{\min (1 / d, d)}{1+d}} \sqrt{\delta}} \leq \rho_{s, m} \leq C \frac{1}{1+\sqrt{\frac{\min (1 / d, d)}{1+d}} \sqrt{\delta}}, \quad m \geq N .
$$

Then it leads to

$$
\begin{aligned}
\sum_{m \geq N}\left(q_{1,2 m}+q_{1,2 m+1}\right) & \simeq\left(q_{1,2 N}+q_{1,2 N+1}\right) \sum_{j=1}^{\infty}\left(\frac{1}{1+\sqrt{\frac{\min (1 / d, d)}{1+d}} \sqrt{\delta}}\right)^{j} \\
& \simeq\left(q_{1,2 N}+q_{1,2 N+1}\right) \frac{1}{\sqrt{\frac{\min (1 / d, d)}{1+d}} \sqrt{\delta}} \\
& \simeq \frac{d}{d+1} \frac{1}{N} \frac{1}{\sqrt{\frac{\min (1 / d, d)}{1+d}} \sqrt{\delta}} \\
& \simeq \max (d, 1) .
\end{aligned}
$$

Therefore, we obtain (21), and replacing $d$ by $\frac{1}{d}$ we also have (22).

\subsubsection{Estimates for $Q_{s}$}

We consider $Q_{1}=\sum_{m=0}^{\infty}\left(q_{1,2 m}-q_{1,2 m+1}\right)$. From definition, $q_{1, m}$ has the decreasing property as

$$
\begin{aligned}
q_{1,2 m+1} & =\left(\rho_{1,2 m+1}\right)\left(q_{1,2 m}\right)<q_{1,2 m}, \\
q_{1,2 m} & =\left(\rho_{1,2 m}\right)\left(q_{1,2 m-1}\right)<q_{1,2 m-1},
\end{aligned}
$$

and therefore

$$
0<\sum_{m \geq N}\left(q_{1,2 m}-q_{1,2 m+1}\right)<q_{1,2 N} \leq C \frac{d}{(d+1)(N+1)} .
$$

This means that $\sum_{m>N}\left(q_{1,2 m}-q_{1,2 m+1}\right)$ shrinks to 0 as $\delta$ goes to 0 .

On the other hand, it follows from (35) and (36) that

$$
\begin{aligned}
\sum_{0 \leq m<N}\left(q_{1,2 m}-q_{1,2 m+1}\right) & =\sum_{m<N} q_{1,2 m}\left(1-\rho_{1,2 m+1}\right) \\
& =\left[\sum_{m<N} \frac{d}{(m+1)(d+1)(m(d+1)+d)}\right]\left(1+N \sqrt{\frac{d+1}{d}} O(\sqrt{\delta})\right) .
\end{aligned}
$$


Taking an advantage of a strict decreasing sequence, we get

$$
\frac{1}{2}\left(\frac{1}{d+1}+\mathfrak{A}\right)<\sum_{0 \leq m<N} \frac{d}{(m+1)(d+1)(m(d+1)+d)}<\frac{1}{d+1}+\mathfrak{A},
$$

where

$$
\begin{aligned}
\mathfrak{A} & =\int_{0}^{M} \frac{d}{(t+1)(d+1)(t(d+1)+d)} d t \\
& =\frac{d}{d+1} \int_{0}^{N}\left[\frac{1}{t+\frac{d}{d+1}}-\frac{1}{t+1}\right] d t \\
& =\frac{d}{d+1}\left[\log \frac{d+1}{d}+\log \left(\frac{N+\frac{d}{d+1}}{N+1}\right)\right] .
\end{aligned}
$$

As has been mentioned, $\delta$ is assumed to be small enough so that

$$
0 \leq-\log \left(\frac{N+\frac{d}{d+1}}{N+1}\right) \leq \frac{1}{2} \log \frac{d+1}{d} .
$$

It leads to

$$
\frac{1}{4} \frac{d}{d+1}\left(\log \frac{d+1}{d}+\frac{1}{d}\right) \leq Q_{1} \leq \frac{3}{2} \frac{d}{d+1}\left(\log \frac{d+1}{d}+\frac{1}{d}\right) .
$$

Note that

$$
\log (1+x)<x, \quad \text { for } x>0 \text {. }
$$

Therefore, we obtain

$$
\frac{1}{C_{1}} \frac{1}{d+1} \leq Q_{1} \leq C_{1} \frac{1}{d+1} .
$$

Similarly, replacing $d$ by $\frac{1}{d}$, we also have

$$
\frac{1}{C_{2}} \frac{d}{d+1} \leq Q_{2} \leq C_{2} \frac{d}{d+1} .
$$

\subsubsection{Estimates for $\sum_{k=0}^{\infty}\left(c_{s, 2 k}\right)\left(q_{s, 2 k}\right)-\sum_{k=0}^{\infty}\left(c_{s, 2 k+1}\right)\left(q_{s, 2 k+1}\right)$}

The lower and upper bounds of

$$
\sum_{m=0}^{\infty}\left(c_{s, 2 m}\right)\left(q_{s, 2 m}\right)-\sum_{m=0}^{\infty}\left(c_{s, 2 m+1}\right)\left(q_{s, 2 m+1}\right), \quad s=1,2
$$

are established here. From (27), (31), (32) and (37), we calculate

$$
\begin{aligned}
\frac{1}{r_{1}} \sum_{m=0}^{\infty}\left(c_{1,2 m}\right)\left(q_{1,2 m}\right) & =\sum_{0 \leq m \leq N}\left(y_{1,2 m}\right)\left(q_{1,2 m}\right)+\sum_{m>N}\left(y_{1,2 m}\right)\left(q_{1,2 m}\right) \\
& =\sum_{0 \leq m \leq N} C\left(\frac{d}{m(d+1)+d}\right)^{2}+\sqrt{\frac{d+1}{d}} O(\sqrt{\delta}) \sum_{m>N} q_{1,2 m} \\
& \leq C .
\end{aligned}
$$


Moreover,

$$
\frac{1}{r_{1}} \sum_{k=0}^{\infty}\left(c_{1,2 k}\right)\left(q_{1,2 k}\right) \geq\left(y_{1,0}\right)\left(q_{1,0}\right)=1
$$

Similarly, we have

$$
\left(y_{1,1}\right)\left(-q_{1,1}\right) \leq-\frac{1}{r_{1}} \sum_{k=0}^{\infty}\left(c_{1,2 k+1}\right)\left(q_{1,2 k+1}\right) \leq C,
$$

and, in total, we have

$$
\frac{1}{C} \leq \frac{1}{r_{1}}\left[\sum_{k=0}^{\infty}\left(c_{1,2 k}\right)\left(q_{1,2 k}\right)-\sum_{k=0}^{\infty}\left(c_{1,2 k+1}\right)\left(q_{1,2 k+1}\right)\right] \leq C .
$$

By the same way,

$$
\frac{1}{C} \leq-\frac{1}{r_{2}}\left[\sum_{k=0}^{\infty}\left(c_{2,2 k}\right)\left(q_{2,2 k}\right)-\sum_{k=0}^{\infty}\left(c_{2,2 k+1}\right)\left(q_{2,2 k+1}\right)\right] \leq C .
$$

\subsection{The Derivation for Theorem 2.3}

Lemma 4.5 Assume that the dimension $n \geq 4$ and the distance $\epsilon$ is sufficiently small. Then, there is a positive constant $C$ independent of $r_{1}, r_{2}$, and $\epsilon$ satisfying the following properties:

- Estimates for $\sum_{m=0}^{\infty} q_{s, m}^{n-2}$ :

$$
\sum_{m=0}^{\infty} q_{1, m}^{n-2} \simeq 1 \text { and } \sum_{m=0}^{\infty} q_{2, m}^{n-2} \simeq 1
$$

where $d=\frac{r_{2}}{r_{1}}$.

- Estimates for $Q_{s}$ :

$$
Q_{1} \simeq \frac{1}{d+1} \text { and } Q_{2} \simeq \frac{d}{d+1}
$$

where $Q_{1}$ and $Q_{2}$ are defined in Lemma 4.1.

- Estimates for $\sum_{k=0}^{\infty}\left(c_{s, 2 k}\right)\left(q_{s, 2 k}\right)-\sum_{k=0}^{\infty}\left(c_{s, 2 k+1}\right)\left(q_{s, 2 k+1}\right)$ :

$$
(-1)^{s+1}\left[\sum_{k=0}^{\infty}\left(c_{s, 2 k}\right)\left(q_{s, 2 k}\right)^{n-2}-\sum_{k=0}^{\infty}\left(c_{s, 2 k+1}\right)\left(q_{s, 2 k+1}\right)^{n-2}\right] \simeq r_{s} \text { for } s=1,2 .
$$

Proof. Let $N$ be as chosen in the proof of Lemma 4.2. We have shown in Estimates for $\sum_{m=0}^{\infty} q_{s, m}$ that for $j=1,2$,

$$
q_{1,2 m+j} \simeq \frac{d}{d+1} \frac{1}{m+1} \quad \text { for } 1 \leq m \leq N
$$


and $q_{1,0}=1$. This yields

$$
\sum_{0 \leq m \leq N} q_{s, m}^{n-2} \simeq 1
$$

and by the argument of (37), we have

$$
\begin{aligned}
\sum_{m \geq N}\left(q_{1,2 m}^{n-2}+q_{1,2 m+1}^{n-2}\right) & \simeq\left(q_{1,2 N}^{n-2}+q_{1,2 N+1}^{n-2}\right) \sum_{j=1}^{\infty}\left(\frac{1}{1+\sqrt{\frac{\min (1 / d, d)}{1+d}} \sqrt{\delta}}\right)^{j(n-2)} \\
& \simeq\left(q_{1,2 N}^{n-2}+q_{1,2 N+1}^{n-2}\right) \frac{1}{\sqrt{\frac{\min (1 / d, d)}{1+d}} \sqrt{\delta}} \\
& \simeq\left(\frac{d}{d+1} \frac{1}{N}\right)^{n-2} \frac{1}{\sqrt{\frac{\min (1 / d, d)}{1+d}} \sqrt{\delta}} .
\end{aligned}
$$

Since $N$ increase at the rate of $\frac{1}{\sqrt{\delta}}$ as $\delta$ goes to zero, $\sum_{m \geq N}\left(q_{1,2 m}^{n-2}+q_{1,2 m+1}^{n-2}\right)$ shrinks to zero as $\delta$ goes to zero. Therefore, we obtain $\sum_{m=0}^{\infty} q_{1, m}^{n-2} \simeq 1$, and similarly $\sum_{m=0}^{\infty} q_{2, m}^{n-2} \simeq 1$.

Now we consider $Q_{s}=\sum_{m=0}^{\infty}(-1)^{m}\left(q_{s, m}\right)^{n-2}$ for $s=1,2$. We note that the tail sum

$$
\left|\sum_{m \geq 2 N}(-1)^{m}\left(q_{1, m}\right)^{n-2}\right| \leq\left|\sum_{m \geq N} q_{1,2 m}^{n-2}+q_{1,2 m+1}^{n-2}\right|
$$

and the upper bound in the right hand side has been shown above to shrink to zero as $\delta$ goes to zero. In this respect, we estimate only $\sum_{m \leq 2 N-1}(-1)^{m}\left(q_{1, m}\right)^{n-2}$ for $Q_{1}$. From definition, we have

$$
\begin{aligned}
\sum_{m \leq 2 N-1}(-1)^{m}\left(q_{1, m}\right)^{n-2} & =\sum_{m \leq N}\left(q_{1,2 m}\right)^{n-2}\left(1-\left(\rho_{2,2 m+1}\right)^{n-2}\right) \\
& \simeq \sum_{m \leq N}\left(\frac{d}{m(d+1)+d}\right)^{n-2}\left(1-\rho_{2,2 m+1}\right) \\
& \simeq \sum_{m \leq N}\left(\frac{d}{m(d+1)+d}\right)^{n-2}\left(\frac{1}{(m+1)(d+1)}+\frac{1}{\sqrt{d(d+1)}} O(\sqrt{\delta})\right) \\
& \simeq \sum_{m \leq N}\left(\frac{d}{m(d+1)+d}\right)^{n-2}\left(\frac{1}{(m+1)(d+1)}\right) \\
& \simeq \frac{1}{d+1}+\sum_{1<m \leq N} \frac{d^{n-2}}{(d+1)^{n-1}(m+1)^{n-2}} \\
& \simeq \frac{1}{d+1} .
\end{aligned}
$$

Therefore, we obtain $Q_{1} \simeq \frac{1}{d+1}$, and replacing $d$ by $\frac{1}{d}$, also have $Q_{2} \simeq \frac{d}{d+1}$. 
We consider the last estimate. By Lemma 4.2 we have

$$
\begin{aligned}
1 & \leq \frac{c_{1,0}}{r_{1}} q_{1,0}^{n-1} \\
& \leq \frac{1}{r_{1}}\left[\sum_{k=0}^{\infty}\left(c_{1,2 k}\right)\left(q_{1,2 k}\right)^{n-2}-\sum_{k=0}^{\infty}\left(c_{1,2 k+1}\right)\left(q_{1,2 k+1}\right)^{n-2}\right] \\
& \leq \frac{1}{r_{1}}\left[\sum_{k=0}^{\infty}\left(c_{1,2 k}\right)\left(q_{1,2 k}\right)-\sum_{k=0}^{\infty}\left(c_{1,2 k+1}\right)\left(q_{1,2 k+1}\right)\right] \simeq 1 .
\end{aligned}
$$

Therefore, we have

$$
\left[\sum_{k=0}^{\infty}\left(c_{1,2 k}\right)\left(q_{1,2 k}\right)^{n-2}-\sum_{k=0}^{\infty}\left(c_{1,2 k+1}\right)\left(q_{1,2 k+1}\right)^{n-2}\right] \simeq r_{1}
$$

and similarly

$$
-\left[\sum_{k=0}^{\infty}\left(c_{2,2 k}\right)\left(q_{2,2 k}\right)^{n-2}-\sum_{k=0}^{\infty}\left(c_{2,2 k+1}\right)\left(q_{2,2 k+1}\right)^{n-2}\right] \simeq r_{2}
$$

\subsubsection{The proof of Theorem 2.3}

We first consider the case of $H\left(x_{1}, x_{2}, \cdots, x_{n}\right)=x_{1}$. Then, Lemma 4.1 implies

$$
\begin{aligned}
\left.u\right|_{\partial D_{1}}-\left.u\right|_{\partial D_{2}}= & \frac{Q_{2}}{M} \sum_{k=0}^{\infty}\left(c_{1,2 k}\right)\left(q_{1,2 k}\right)^{n-2}+\frac{Q_{1}}{M} \sum_{k=0}^{\infty}\left(c_{2,2 k+1}\right)\left(q_{2,2 k+1}\right)^{n-2} \\
& -\frac{Q_{2}}{M} \sum_{k=0}^{\infty}\left(c_{1,2 k+1}\right)\left(q_{1,2 k+1}\right)^{n-2}-\frac{Q_{1}}{M} \sum_{k=0}^{\infty}\left(c_{2,2 k}\right)\left(q_{2,2 k}\right)^{n-2} .
\end{aligned}
$$

where $c_{s, j}$ is the $x_{1}$-coordinate of $\mathbf{c}_{s, j}$ for $s=1,2, j \in \mathbb{N}$, i.e.,

$$
\left(c_{s, j}, 0, \cdots, 0\right)=\mathbf{c}_{s, j} .
$$

Lemma 4.5 allows one to estimate four positive valued terms in the right hand side of (39) so that

$$
\begin{aligned}
M & =Q_{2} \sum_{k=0}^{\infty} q_{1,2 k}+Q_{1} \sum_{k=0}^{\infty} q_{2,2 k+1} \\
& \simeq 1
\end{aligned}
$$

In total, we obtain

$$
|u|_{\partial D_{1}}-\left.u\right|_{\partial D_{2}} \mid \simeq \frac{r_{1} r_{2}}{r_{1}+r_{2}} .
$$


In the case of $H=\sum_{i=2}^{n} a_{i} x_{i}$, the integration (7) is zero, because all point charges of $h$ lie on $x_{1}$ axis. Thus, there is no potential difference between inclusions. Therefore, we have established the estimate for the potential difference between $D_{1}$ and $D_{2}$.

Now, we consider the upper bound of $|\nabla u|$ when $H(\mathbf{x})=\sum_{i=1}^{n} a_{i} x_{i}$. To do so, we pursue the argument similar to Theorem 2.1. We thus assume that $r_{1} \geq r_{2}$. Let $\frac{1}{r_{1}} D_{1}$ and $\frac{1}{r_{1}} D_{2}$ be the same as defined in the proof of Theorem 2.1. We choose the sphere $B_{4}(0, \ldots, 0)$ as the domain $\Omega$ containing $D_{1}$ and $D_{2}$ independently of any small distance $\epsilon>0$. We consider

$$
\widetilde{u}(\mathbf{x}):=\frac{1}{r_{1}} u\left(r_{1} \mathbf{x}\right) .
$$

Then, $\widetilde{u}$ is the solution to (11) for $H(\mathbf{x})=\sum_{i=1}^{3} a_{i} x_{i}$ when the inclusions are $\frac{1}{r_{1}} D_{1}$ and $\frac{1}{r_{1}} D_{2}$ instead of $D_{1}$ and $D_{2}$. It follows from the estimate for the difference of $u$ between $D_{1}$ and $D_{2}$ that

$$
|\widetilde{u}|_{\partial\left(\frac{1}{r_{1}} D_{1}\right)}-\left.\widetilde{u}\right|_{\partial\left(\frac{1}{r_{1}} D_{2}\right)}|\simeq| a_{1} \mid \frac{d}{1+d},
$$

where $d=\frac{r_{2}}{r_{1}}$ and $\delta=\frac{\epsilon}{r_{1}}$. As defined to (0.9) in [4, $v_{3}$ is defined as follows:

$$
\begin{cases}\Delta v_{3}=0 & \text { in } \Omega \backslash \overline{\left(\frac{1}{r_{1}} D_{1} \cup \frac{1}{r_{1}} D_{2}\right)} \\ v_{3}=0 & \text { on } \partial\left(\frac{1}{r_{1}} D_{1} \cup \frac{1}{r_{1}} D_{2}\right) \\ v_{3}=-\widetilde{u} & \text { on } \partial \Omega\end{cases}
$$

Lemma 4.3 and 4.4 have been used in the proof of Theorem 2.1. By the help of them, we can obtain

$$
\left\|\nabla\left(\widetilde{u}+v_{3}\right)\right\|_{L^{\infty}\left(\Omega \backslash\left(\frac{1}{r_{1}} D_{1} \cup \frac{1}{r_{1}} D_{2}\right)\right)} \leq C\left|a_{1}\right|\left(\frac{d}{1+d}\right) \frac{1}{|\delta|} .
$$

and

$$
\left\|\nabla v_{3}\right\|_{L^{\infty}\left(\partial\left(\frac{1}{r_{1}} D_{1} \cup \frac{1}{r_{1}} D_{2}\right)\right)} \leq C \frac{1}{d}\left(\left|a_{1}\right|\left(\frac{d}{1+d}\right)+\sum_{i=1}^{n}\left|a_{i}\right|\right) .
$$

Two bounds lead to

$$
\begin{aligned}
\|\nabla u\|_{L^{\infty}\left(\partial\left(D_{1} \cup D_{2}\right)\right)} & =\|\nabla \widetilde{u}\|_{L^{\infty}\left(\partial\left(\frac{1}{r_{1}} D_{1} \cup \frac{1}{r_{1}} D_{2}\right)\right)} \\
& \leq C\left(\left|a_{1}\right|\left(\frac{d}{1+d}\right) \frac{1}{|\delta|}+\frac{1}{d} \sum_{i=2}^{n}\left|a_{i}\right|\right) \\
& \leq C^{\prime}\left(\left|a_{1}\right|\left(\frac{r_{1} r_{2}}{r_{1}+r_{2}}\right) \frac{1}{|\epsilon|}+\frac{1}{d} \sum_{i=2}^{n}\left|a_{i}\right|\right)
\end{aligned}
$$

for any sufficiently small $\epsilon>0$. Since $|\nabla H|$ is bounded by $\sum_{i=1}^{n}\left|a_{i}\right|$, the harmonicity of $u-H$ in $\mathbb{R}^{n} \backslash \Omega$ leads to

$$
\|\nabla u\|_{L^{\infty}\left(\mathbb{R}^{n} \backslash\left(D_{1} \cup D_{2}\right)\right)} \leq C\left(\left|a_{1}\right|\left(\frac{r_{1} r_{2}}{r_{1}+r_{2}}\right) \frac{1}{|\epsilon|}+\frac{1}{d} \sum_{i=2}^{n}\left|a_{i}\right|\right) .
$$

Therefore, we obtain the upper bound of the gradient estimate. In addition, the lower bound of the gradient is immediately derived from the mean value theorem. 


\subsection{The proof for Theorem 2.4}

Without the loss of generality, we may assume that $H(0, \cdots, 0)=0$. Then, Lemma 4.1 implies

$$
\begin{aligned}
|u|_{\partial D_{1}}-\left.u\right|_{\partial D_{2}} \mid= & \mid \frac{Q_{2}}{M} \sum_{k=0}^{\infty} H\left(\mathbf{c}_{1,2 k}\right)\left(q_{1,2 k}\right)^{n-2}+\frac{Q_{1}}{M} \sum_{k=0}^{\infty} H\left(\mathbf{c}_{2,2 k+1}\right)\left(q_{2,2 k+1}\right)^{n-2} \\
& \quad-\frac{Q_{2}}{M} \sum_{k=0}^{\infty} H\left(\mathbf{c}_{1,2 k+1}\right)\left(q_{1,2 k+1}\right)^{n-2}-\frac{Q_{1}}{M} \sum_{k=0}^{\infty} H\left(\mathbf{c}_{2,2 k}\right)\left(q_{2,2 k}\right)^{n-2} \mid \\
\leq & \|\nabla H\|_{L^{\infty}(\Omega)} \mid \frac{Q_{2}}{M} \sum_{k=0}^{\infty}\left(c_{1,2 k}\right)\left(q_{1,2 k}\right)^{n-2}+\frac{Q_{1}}{M} \sum_{k=0}^{\infty}\left(c_{2,2 k+1}\right)\left(q_{2,2 k+1}\right)^{n-2} \\
& \quad-\frac{Q_{2}}{M} \sum_{k=0}^{\infty}\left(c_{1,2 k+1}\right)\left(q_{1,2 k+1}\right)^{n-2}-\frac{Q_{1}}{M} \sum_{k=0}^{\infty}\left(c_{2,2 k}\right)\left(q_{2,2 k}\right)^{n-2} \mid
\end{aligned}
$$

Owing to Lemma 4.2 and 4.5 we can choose a constant $C$ independent of $\epsilon, r_{1}, r_{2}$ and $\|\nabla H\|_{L^{\infty}(\Omega)}$ satisfying

$$
|u|_{\partial D_{1}}-\left.u\right|_{\partial D_{2}} \mid \leq C\|\nabla H\|_{L^{\infty}(\Omega)}\left(\frac{r_{1} r_{2}}{r_{1}+r_{2}}\right) \cdot \begin{cases}\frac{1}{\mid \log \epsilon} & \text { if } n=3 \\ 1 & \text { if } n \geq 4\end{cases}
$$

Here, we would establish the upper bound of the gradient. The method is the same as presented in the proofs of Theorem 2.1 and 2.3. We thus assume that $r_{1} \geq r_{2}$. Let $\frac{1}{r_{1}} D_{i}(i=1,2), \widetilde{u}, d$ and $\delta$ be as defined in Theorem 2.1 and 2.3. Then $\widetilde{u}$ is the solution to (11) for

$$
\widetilde{H}:=\frac{1}{r_{1}} H\left(r_{1} \mathbf{x}\right)
$$

instead of $H$, replacing $D_{i}$ by $\frac{1}{r_{1}} D_{i}$ for $i=1,2$. We consider $\frac{1}{r_{1}} \Omega:=\left\{\mathbf{x} \in \mathbb{R}^{n} \mid r_{1} \mathbf{x} \in \Omega\right\}$, because $\Omega$ is the ball $B_{4\left(r_{1}+r_{2}\right)}(0, \cdots, 0)$ in this theorem. So, the radius of the ball $\frac{1}{r_{1}} \Omega$ is between 4 and 8 . Note that

$$
\|\nabla \widetilde{H}\|_{L^{\infty}(\Omega)}=\|\nabla H\|_{L^{\infty}\left(\frac{1}{r_{1}} \Omega\right)} .
$$

We thus choose the harmonic function $v_{3}$ satisfying the same definition as Theorem 2.1 and 2.3. replacing $\Omega$ by $\frac{1}{r_{1}} \Omega$. Similarly to Theorem 2.1 and 2.3 , owing to Lemma 4.3 and 4.4 or the lemmas presented in 4, we obtain

$$
\left\|\nabla\left(\widetilde{u}+v_{3}\right)\right\|_{L^{\infty}\left(\frac{1}{r_{1}} \Omega \backslash\left(\frac{1}{r_{1}} D_{1} \cup \frac{1}{r_{1}} D_{2}\right)\right)} \leq C\|\nabla H\|_{L^{\infty}(\Omega)}\left(\frac{d}{1+d}\right) \cdot \begin{cases}\frac{1}{\delta \log \delta \mid} & \text { if } n=3 \\ \frac{1}{\delta} & \text { if } n \geq 4\end{cases}
$$

and

$$
\left\|\nabla v_{3}\right\|_{L^{\infty}\left(\partial\left(\frac{1}{r_{1}} D_{1} \cup \frac{1}{r_{1}} D_{2}\right)\right)} \leq C \frac{1}{d}\|\nabla H\|_{L^{\infty}(\Omega)}\left(\frac{d}{1+d}+1\right) .
$$

Two bounds above yield

$$
\|\nabla \widetilde{u}\|_{L^{\infty}\left(\partial\left(\frac{1}{r_{1}} D_{1} \cup \frac{1}{r_{1}} D_{2}\right)\right)} \leq C\|\nabla H\|_{L^{\infty}(\Omega)}\left(\frac{d}{1+d}\right) \cdot \begin{cases}\frac{1}{|\delta \log \delta|} & \text { if } n=3 \\ \frac{1}{\delta} & \text { if } n \geq 4\end{cases}
$$


By the harmonicity of $\widetilde{u}-\widetilde{H}$ in $\mathbb{R}^{n} \backslash\left(\frac{1}{r_{1}} \Omega\right)$, we have

$$
\begin{aligned}
\|\nabla(u-H)\|_{L^{\infty}\left(\mathbb{R}^{n} \backslash\left(D_{1} \cup D_{2}\right)\right)} & =\|\nabla(\widetilde{u}-\widetilde{H})\|_{L^{\infty}\left(\mathbb{R}^{n} \backslash\left(\frac{1}{r_{1}} D_{1} \cup \frac{1}{r_{1}} D_{2}\right)\right)} \\
& \leq C\|\nabla H\|_{L^{\infty}(\Omega)}\left(\frac{r_{1} r_{2}}{r_{1}+r_{2}}\right) \cdot \begin{cases}\frac{1}{|\epsilon \log \epsilon|} & \text { if } n=3 \\
\frac{1}{\epsilon} & \text { if } n \geq 4\end{cases}
\end{aligned}
$$

Furthermore, this leads to

$$
\|\nabla u\|_{L^{\infty}\left(\Omega \backslash\left(D_{1} \cup D_{2}\right)\right)} \leq C\|\nabla H\|_{L^{\infty}(\Omega)}\left(\frac{r_{1} r_{2}}{r_{1}+r_{2}}\right) \cdot \begin{cases}\frac{1}{\epsilon \log \epsilon \mid} & \text { if } n=3 \\ \frac{1}{\epsilon} & \text { if } n \geq 4\end{cases}
$$

Therefore, we have completed the proof.

\section{Acknowledgements}

The authors would like to express their gratitude to Professor YanYan Li and his students Dr. Shiting Bao and Biao Yin, who gave them a precious information on the optimal blowup rate in the higher dimensions and also encouraged the second named author to check his result. The authors are also deeply grateful to Professor Hyeonbae Kang, who suggested the original subject studied in this paper and gave useful comments. The second named author would also like to express his thanks to Professor David Kinderlehrer, and gratefully acknowledges his hospitality during the visiting period at Carnegie Mellon University. The first author was supported by the Korea Research Foundation Grant funded by the Korean Goverment (MOEHRD) (KRF-2005-214-C00184).

\section{References}

[1] Ammari H, Dassios G, Kang H, and Lim M, Estimates for the electric field in the presence of adjacent perfectly conducting spheres, Quat. Appl. Math., 65 (2007), pp. 339-355

[2] H. Ammari, H. Kang, And M. Lim, Gradient estimates for solutions to the conductivity problem, Math. Ann., 332(2) (2005), pp. 277-286.

[3] H. Ammari, H. Kang, H. Lee, J. Lee, and M. Lim, Optimal bounds on the gradient of solutions to conductivity problems, J. Math. Pures Appl., 88 (2007), pp. 307-324.

[4] E. Bao, Y.Y. Li and B. Yin Gredient estimats for the conductivity problem, Arch. Rational Mech. Anal, to appear.

[5] B. Budiansky and G. F. Carrier, High shear stresses in stiff fiber composites, J. Appl. Mech., 51 (1984), pp. 733-735.

[6] E. Bonnetier And M. Vogelius, An Elliptic regularity result for a composite medium with "touching" fibers of circular cross-section, SIAM J. Math. Anal., 31, No 3 (2000), pp. 651-677.

[7] J.D. JACKson, Classical Electrodynamics, Third ed., Wiley, New York, NY, 1999. 
[8] J.B. KelleR, Stresses in narrow regions, Trans. ASME J. Appl. Mech., 60 (1993), pp. 1054-1056.

[9] Y.Y. Li AND M. NiREnBerg, Estimates for elliptic system from composite material, Comm. Pure Appl. Math., LVI (2003), pp. 892-925.

[10] Y.Y. Li And M. Vogelius, Gradient estimates for solution to divergence form elliptic equation with discontinuous coefficients, Arch. Rational Mech. Anal., 153 (2000), pp. 91151.

[11] K. Yun, Estimates for electric fields blown up between closely adjacent conductors with arbitrary shape, SIAM J. Appl. Math., 67, No 3 (2007), pp. 714-730.

[12] K. Yun, Optimal bound on high stresses occurring between stiff fibers with arbitrary shaped cross sections, (2006), submitted. 